\title{
Relations between echocardiography findings and infarct size in patients with ESUS
}

\author{
Kentaro Ishizuka, MD¹; Takao Hoshino, MD, PhD'; Eiko Higuchi, MD¹; Kyomi Ashihara, \\ MD, PhD²; Yuka Shirai, MD¹; Sono Toi, MD,PhD; Nobuhisa Hagiwara, MD, PhD²; Kazuo \\ Kitagawa, MD, PhD ${ }^{1}$
}

1.Department of Neurology, Tokyo Women's Medical University, Japan

2. Department of Cardiology, Tokyo Women's Medical University, Japan

Introduction

A clinical entity termed embolic stroke of undetermined source (ESUS) designates cryptogenic strokes presumably due to embolism with no evidences of lacunar stroke, ipsilateral stenosis in intra- and extracranial arteries, or major cardioembolic sources. The etiologic mechanisms of ESUS are diverse. Transesophageal echocardiography is not essential to diagnose ESUS, however, it is important examination to identify etiopathologenic mechanism. We formulated the hypothesis that the size and the lesion pattern of cerebral infarct are correlated with etiopathologenic mechanism. To verify the hypothesis, we examined the relationship between the size and the lesion pattern of cerebral infarct and echocardiography.

\section{Materials and Methods}

The ethics committee at our institution approved the protocol of this study. TWMU stroke registry (Tokyo Women's Medical University stroke registry)is a prospective, single center registry. This study includes 67 consecutive patients (mean age 62.3 years; male 61.2\%) with ESUS who underwent both transthoracic and transesophageal echocardiography examinations during hospitalization. We classified the infarct size on MRI into three groups; small infarct was defined as $\leq 15 \mathrm{~mm}$ in size, large infarct as $\geq 1 / 3$ of the cerebral hemisphere, and medium infarct as intermediate in size between small and large infarcts. Echocardiographic findings were compared between patients with small and medium/large infarcts. We also classified the lesion pattern. The lesion pattern was assessed on the MRI (Diffusion weighted image) and categorized as single, scattered or multiple territory. Several lesions in only 1 vascular supply territory were defined as scattered lesion pattern. Several lesions in $\geq 2$ vascular supply territory were defined as multiple territory lesion pattern.

Figure 1. Echocardiography characteristics of patients with different type of infarct lesions

Table 2. Clinical characteristics of patients with different infarct size

\begin{tabular}{|c|c|c|c|}
\hline Clinical characteristics & $\begin{array}{c}\text { small infarct } \\
\mathbf{N}=41\end{array}$ & $\begin{array}{c}\text { medium/large } \\
\text { infarct } \\
\mathbf{N}=26\end{array}$ & P value \\
\hline Age, mean \pm SD & $61.9 \pm 13.9$ & $62.8 \pm 14.0$ & 0.78 \\
\hline Men, $\mathrm{n}(\%)$ & $22(53.7)$ & $19(73.1)$ & 0.11 \\
\hline Hypertension, $\mathrm{n}(\%)$ & $27(65.9)$ & $19(73.1)$ & 0.53 \\
\hline Diabetes Mellitus, $\mathrm{n}(\%)$ & $20(48.8)$ & $10(36.5)$ & 0.10 \\
\hline Dyslipidemia, $\mathrm{n}(\%)$ & $15(37.5)$ & $11(42.3)$ & 0.69 \\
\hline Chronic kidney disease, $\mathrm{n}(\%)$ & $11(26.8)$ & $6(23.1)$ & 0.73 \\
\hline Current smoking, $\mathrm{n}(\%)$ & $11(26.8)$ & $8(30.8)$ & 0.82 \\
\hline Current excessive alcohol, $\mathrm{n}(\%)$ & $1(2.4)$ & $5(20.8)$ & 0.05 \\
\hline Previous & $3(7.3)$ & $2(7.7)$ & 0.91 \\
\hline coronary artery disease, $\mathrm{n}(\%)$ & $4(9.8)$ & $6(23.1)$ & 0.17 \\
\hline Previous cerebral infarction, $\mathrm{n}(\%)$ & 14 & 16 \\
\hline NIHSS on admission, & $1(0-3)$ & $2(1-2)$ & 0.21 \\
\hline median (IQR) & $9(22.0)$ & $9(34.6)$ & 0.25 \\
\hline Anti thrombotic usage, $\mathrm{n}(\%)$ & &
\end{tabular}

Table 3. Clinical characteristics of patients with different age

\begin{tabular}{|c|l|l|l|}
\hline & $\begin{array}{l}\text { age } \geqq 60 \\
\mathrm{~N}=40\end{array}$ & $\begin{array}{l}\text { age }<60 \\
\mathrm{~N}=27\end{array}$ & P value \\
\hline Men, $\mathrm{n}(\%)$ & $23(57.5)$ & $18(66.7)$ & 0.45 \\
\hline Hypertension, $\mathrm{n}(\%)$ & $28(70.0)$ & $18(66.7)$ & 0.77 \\
\hline Diabetes Mellitus, $\mathrm{n}(\%)$ & $21(52.5)$ & $9(33.3)$ & 0.12 \\
\hline Dyslipidemia, $\mathrm{n}(\%)$ & $18(46.2)$ & $8(29.6)$ & 0.17 \\
\hline Chronic kidney disease, $\mathrm{n}(\%)$ & $10(25.0)$ & $7(25.9)$ & 0.93 \\
\hline Current smoking, $\mathrm{n}(\%)$ & $8(30.8)$ & $11(28.2)$ & 0.82 \\
\hline Current excessive alcohol, $\mathrm{n}(\%)$ & $2(5.0)$ & $4(14.8)$ & 0.28 \\
\hline Previous coronary artery disease, $\mathrm{n}(\%)$ & $4(10.0)$ & $1(3.7)$ & 0.32 \\
\hline Previous cerebral infarction, $\mathrm{n}(\%)$ & $7(18.4)$ & $3(11.5)$ & 0.45 \\
\hline Small infact on MRI & $25(62.5)$ & $16(59.3)$ & 0.79 \\
\hline Single lesion on MRI & $23(57.7)$ & $15(55.6)$ & 0.87 \\
\hline Modified Rankin scale & $1(1-2)$ & $1(1-1)$ & 0.19 \\
\hline
\end{tabular}

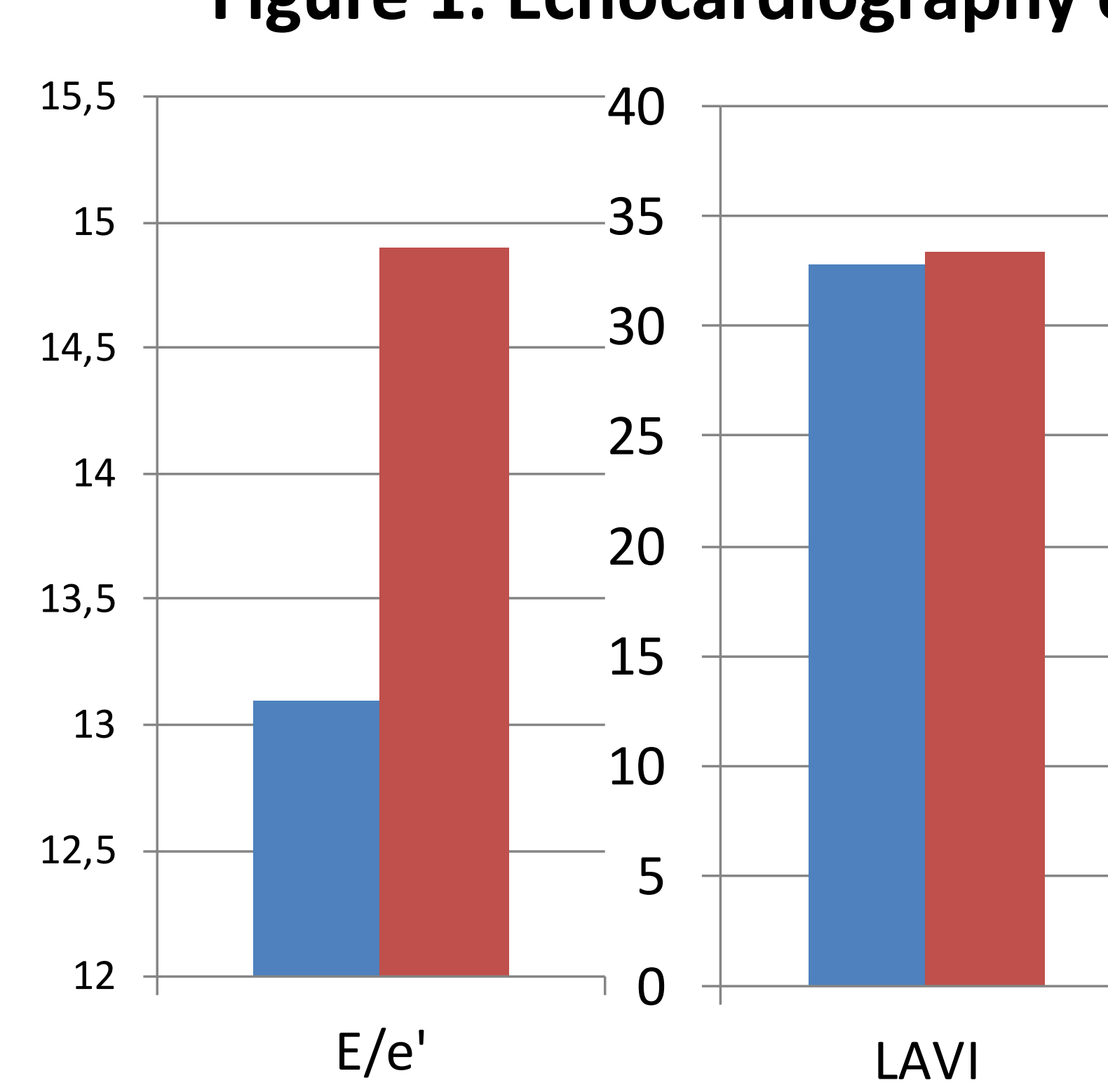

Figure 2. Echocardiography characteristics of patients with different size
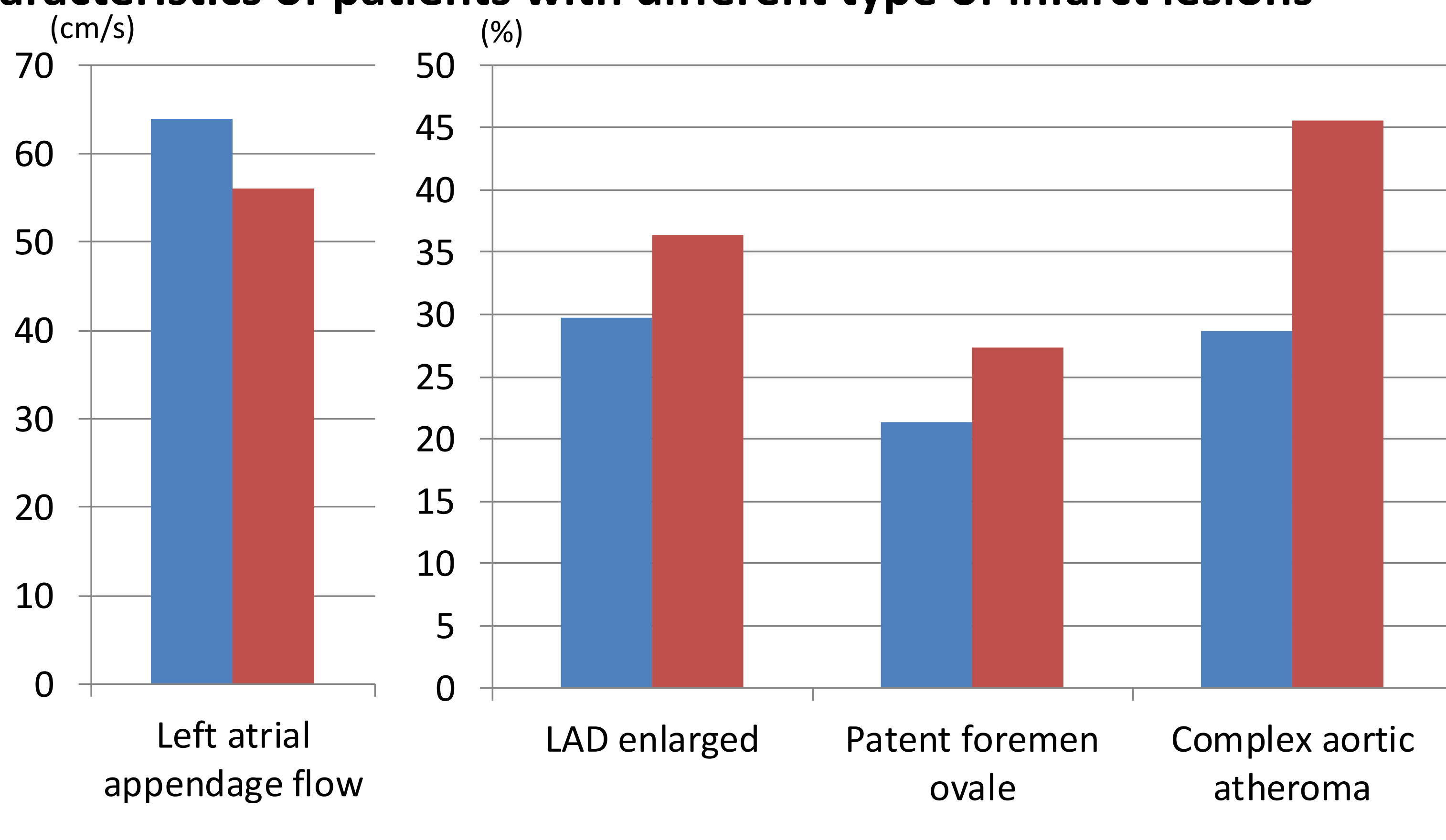

Single
$N=56$

Multiple

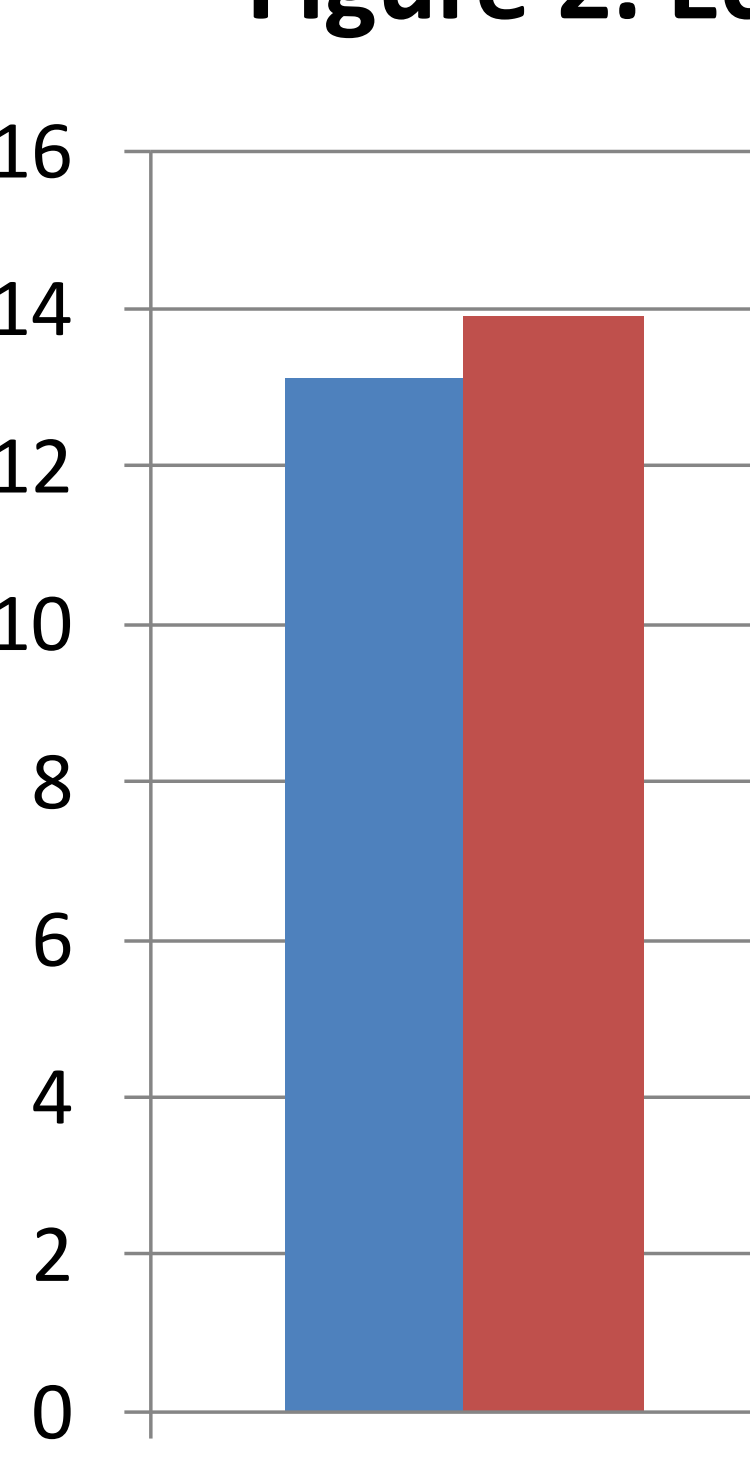

$E / e^{\prime}$

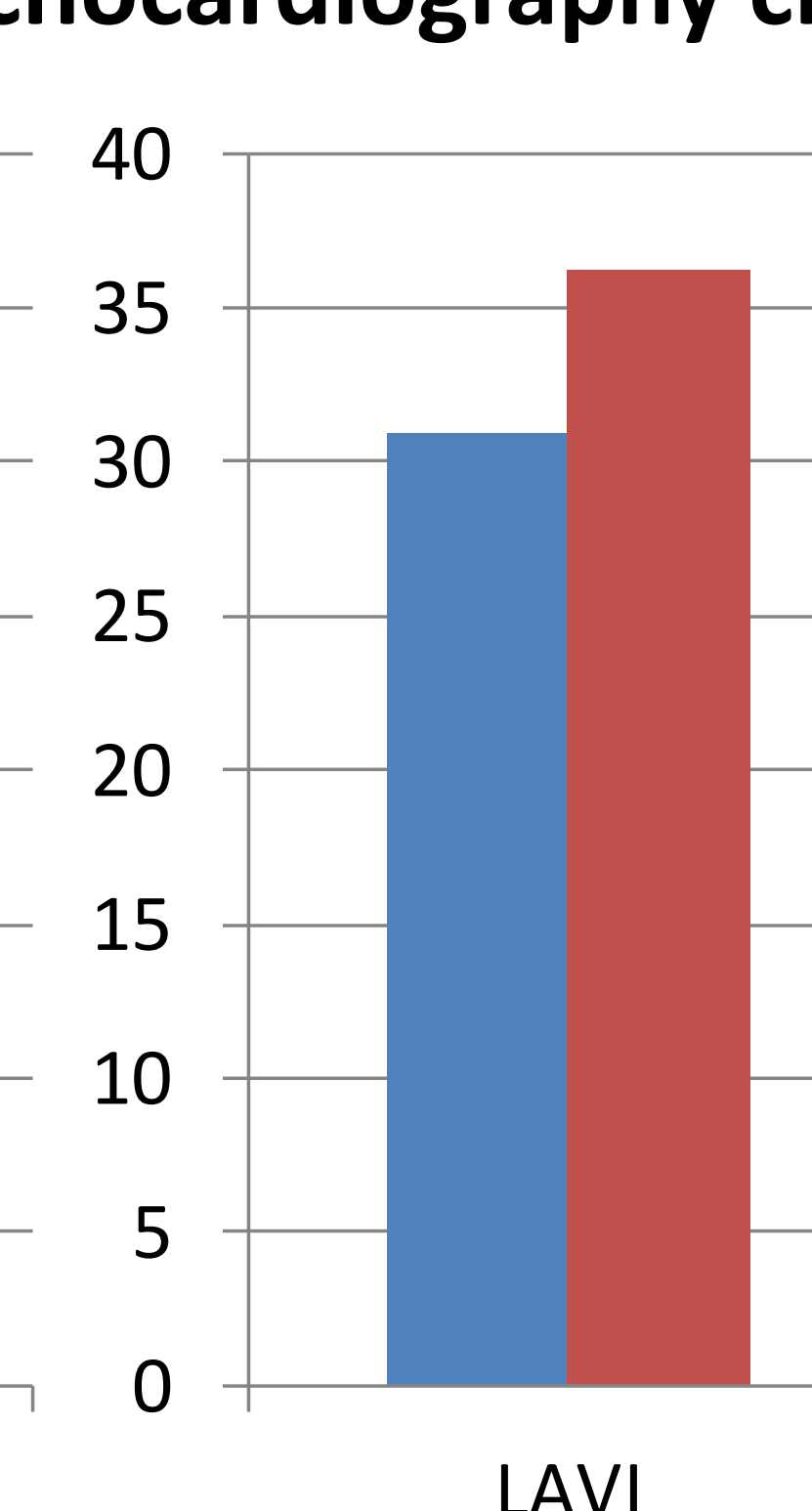

LAVI

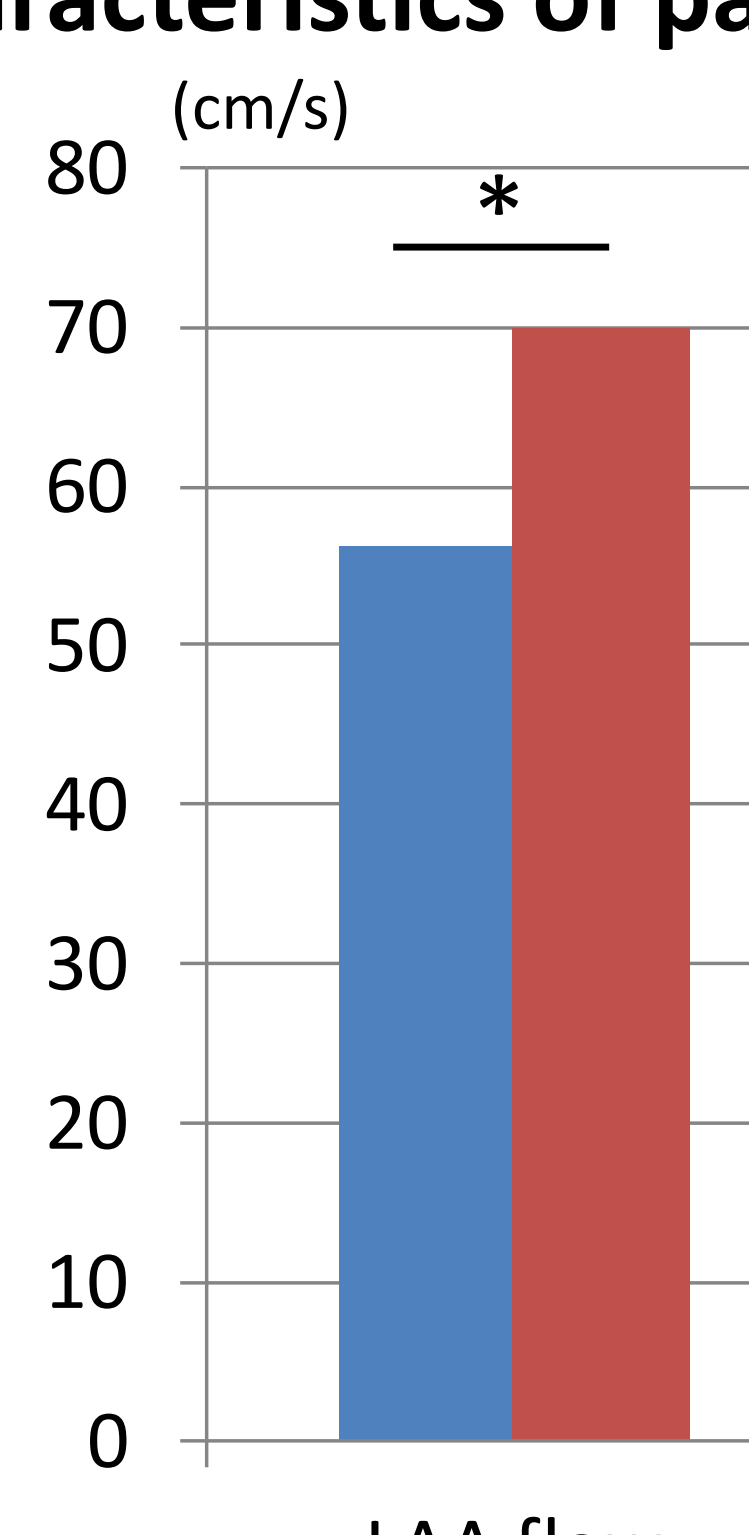

LAA flow

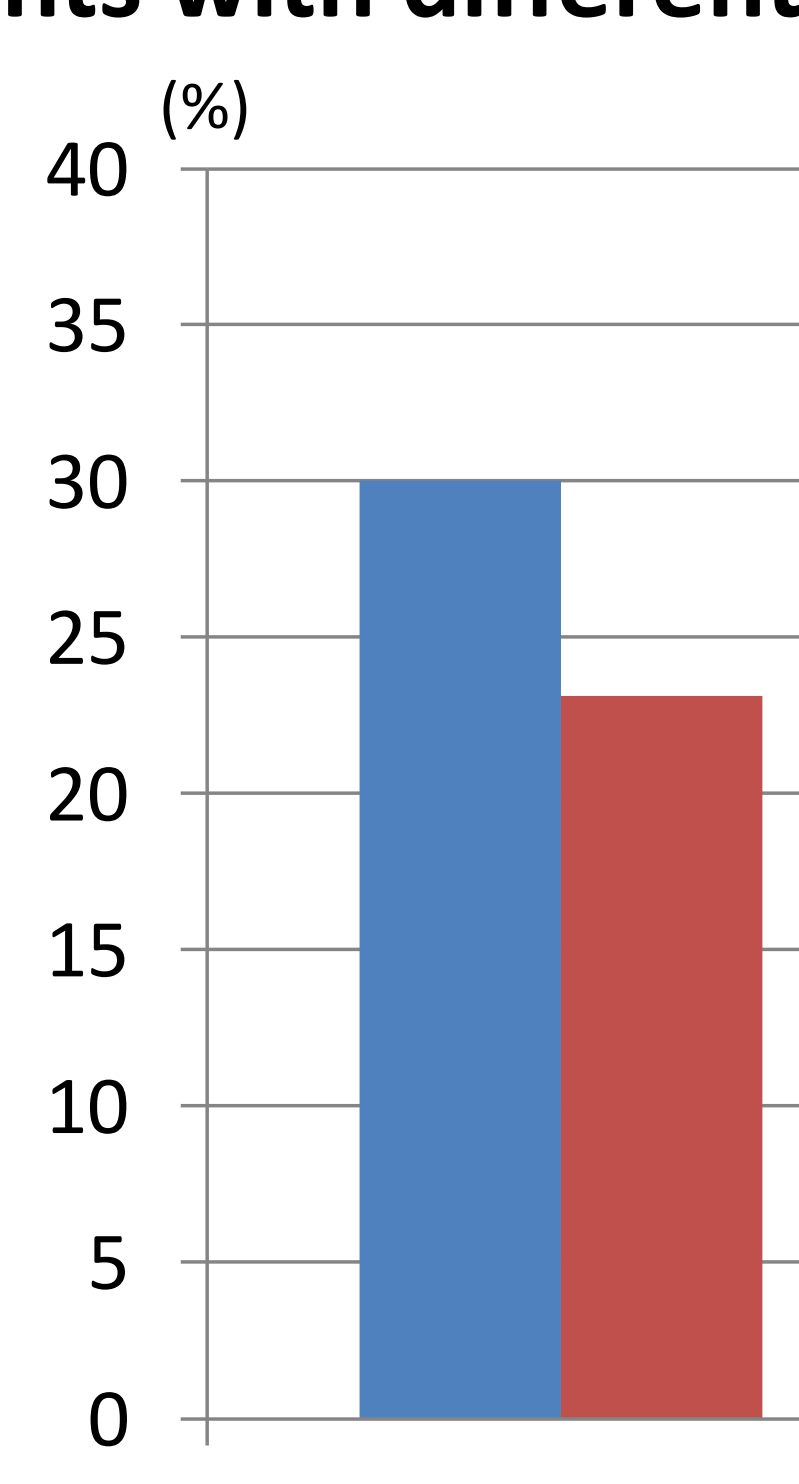

LAD enlarged

\section{Results}

Table 1. Clinical characteristics of patients with different type of infarct lesions

\begin{tabular}{|c|c|c|c|c|}
\hline Clinical characteristics & $\begin{array}{c}\text { Single } \\
\mathrm{N}=38\end{array}$ & $\begin{array}{c}\text { Scattered } \\
\mathrm{N}=18\end{array}$ & $\begin{array}{c}\text { Multiple } \\
\mathrm{N}=11\end{array}$ & P value \\
\hline Age, mean \pm SD & $62.9 \pm 13.6$ & $61.8 \pm 16.3$ & $60.6 \pm 11.0$ & 0.88 \\
\hline Men, $\mathrm{n}(\%)$ & $27(71.1)$ & $10(55.6)$ & $4(36.4)$ & 0.10 \\
\hline Hypertension, $\mathrm{n}(\%)$ & $31(81.6)$ & $9(50.0)$ & $6(54.6)$ & 0.03 \\
\hline Diabetes Mellitus, $\mathrm{n}(\%)$ & $16(42.1)$ & $7(38.9)$ & $7(63.6)$ & 0.37 \\
\hline $\begin{array}{c}\text { Dyslipidemia, } \mathrm{n}(\%) \\
\text { Chronic kidney disease, } \mathrm{n}(\%)\end{array}$ & $18(47.4)$ & $4(23.5)$ & $4(36.4)$ & 0.24 \\
\hline $\begin{array}{c}\text { Current smoking, } \mathrm{n}(\%) \\
\text { Current excessive alcohol, } \mathrm{n}(\%)\end{array}$ & $12(31.6)$ & $3(17.7)$ & $2(18.2)$ & 0.41 \\
\hline $\begin{array}{c}\text { Previous } \\
\text { coronary artery disease, } \mathrm{n}(\%)\end{array}$ & $3(7.9)$ & $3(17.7)$ & $4(36.4)$ & 0.45 \\
\hline $\begin{array}{c}\text { Previous cerebral infarction, } \mathrm{n}(\%) \\
\text { NIHSS on admission, }\end{array}$ & $9(23.7)$ & $1(5.6)$ & 0 & 0.57 \\
\hline median (IQR) & $1(1-2.25)$ & $1(0-2.75)$ & $2(1-3)$ & 0.83 \\
\hline Anti thrombotic usage, $\mathrm{n}(\%)$ & $14(36.8)$ & $3(16.7)$ & $1(9.1)$ & 0.09 \\
\hline
\end{tabular}

Figure 3. Echocardiography characteristics of patients with different age
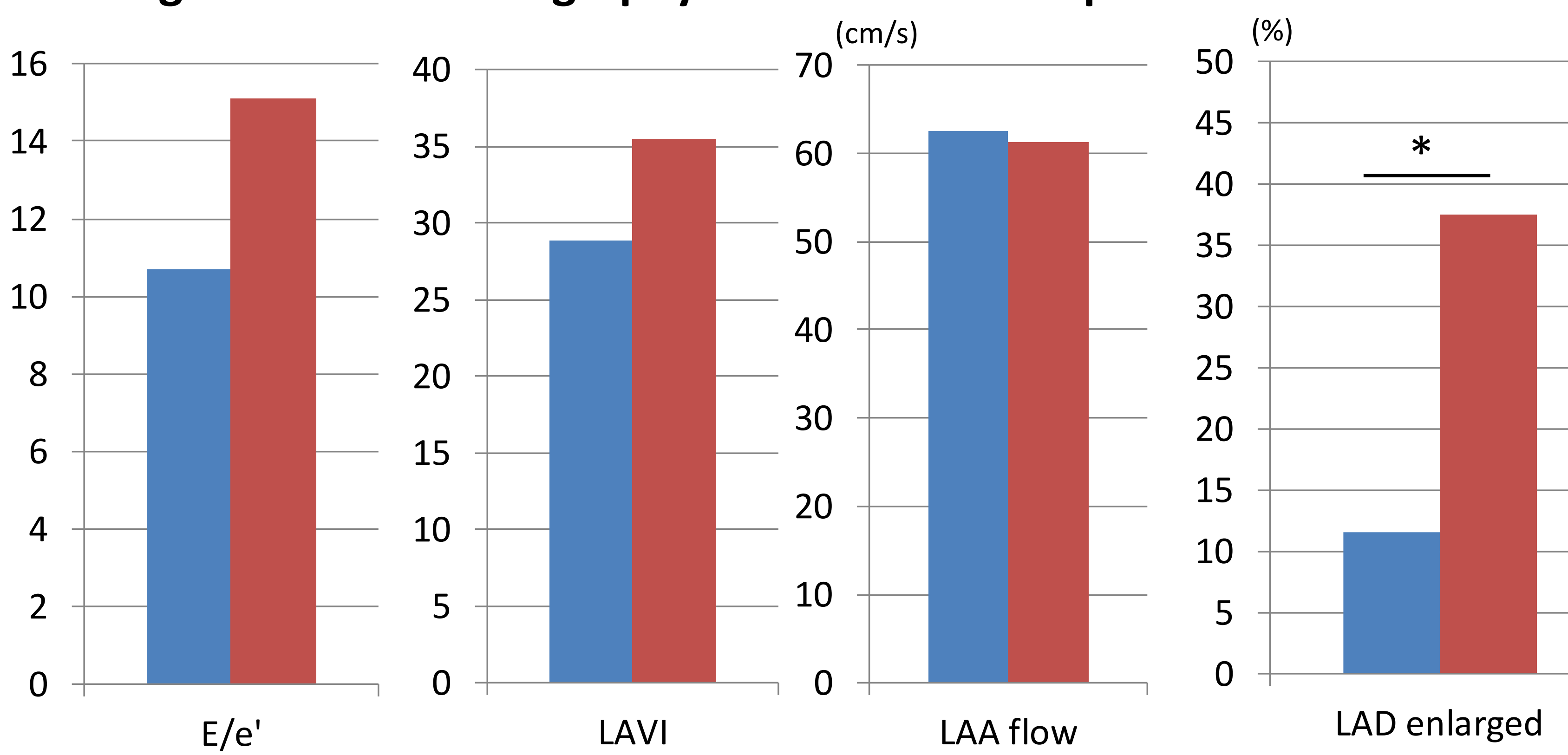
ovale
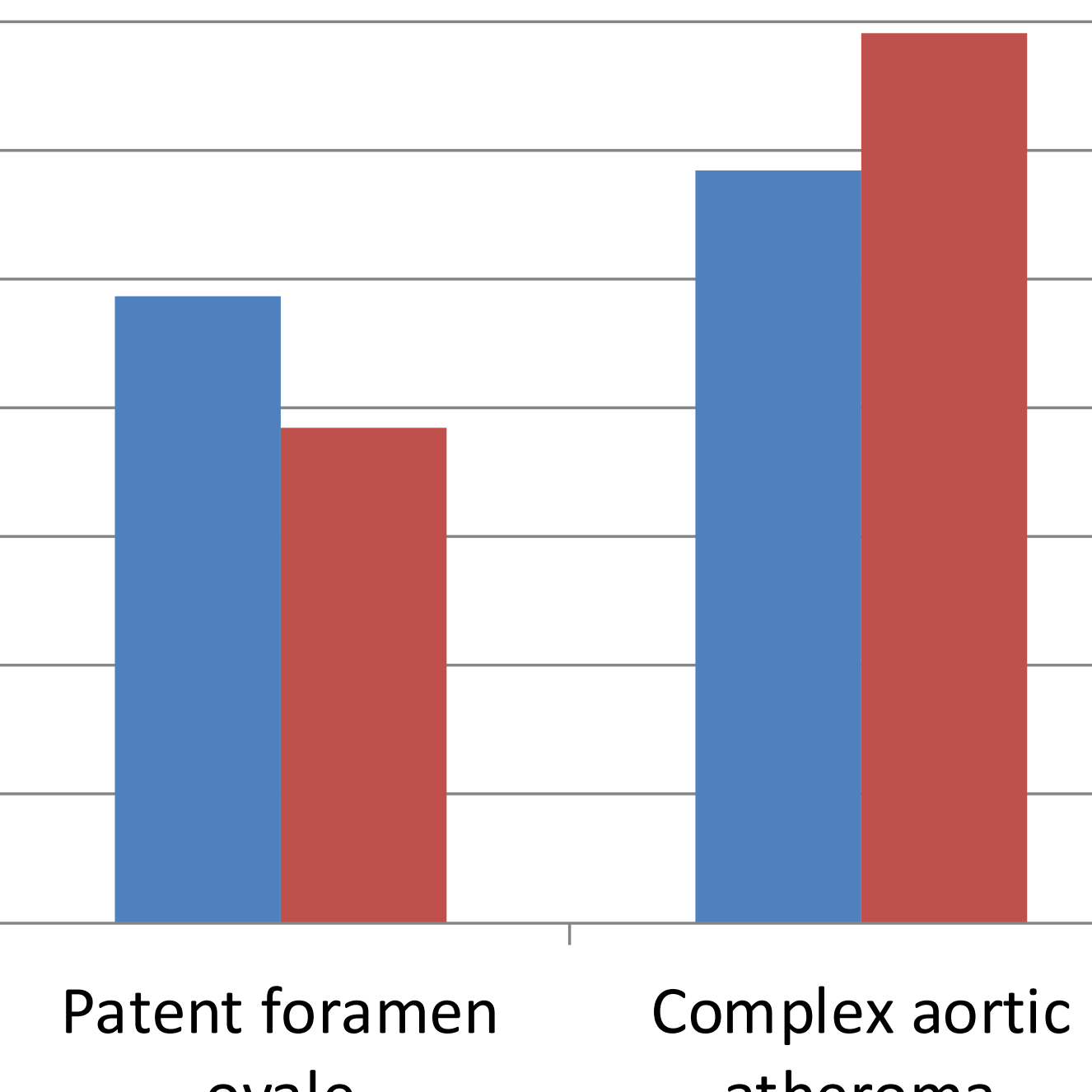
atheroma

LAA flow

LAD enlarged
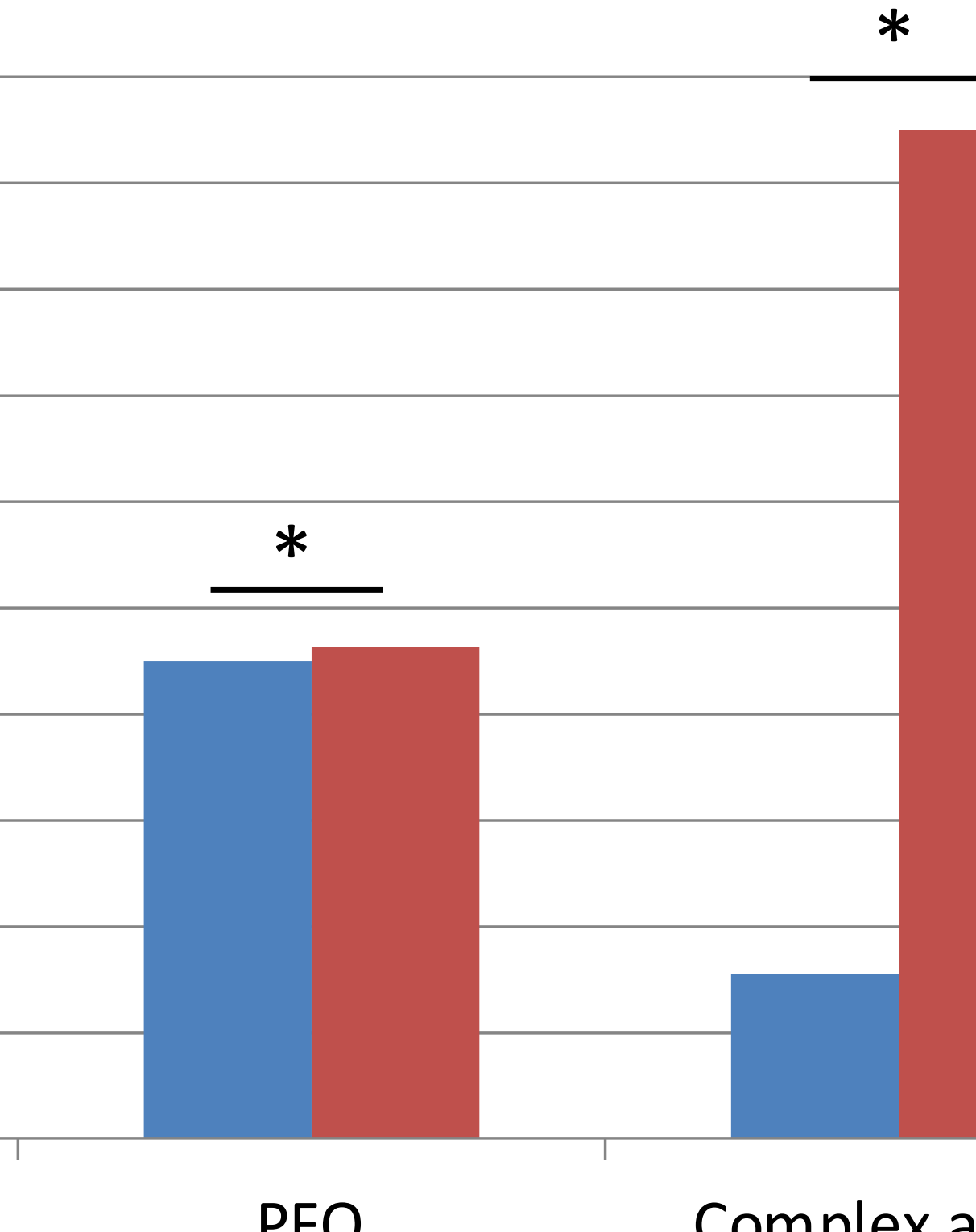

\section{Conclusions}

